\title{
Anestesia para pacientes pediátricos con COVID-19
}

\section{Anesthesia and COVID-19 pediatric patients}

María Francisca Bernucci MD. ${ }^{1}$, Alexandra Fajardo MD. ${ }^{2}$, Verónica Maureira MD. ${ }^{3}$, Rose Marie Heider MD. ${ }^{4}$

\begin{abstract}
Since December 2019, the world has faced the rapid spread of a new virus: SARS-CoV-2. Originating in Wuhan, it has spread quickly to hundreds of countries in the five continents, becoming a pandemia as of March 2020. We confront a highly contagious virus, capable of collapsing healthcare systems. Nearly $2 \%$ of infected individuals worldwide are children. They are usually asymptomatic, thus playing an important role in viral transmission. Less frequently, they can become critically ill. In this article we describe the epidemiology, available to this date, of COVID-19 in the pediatric population. This information will enable to suspect and detect possible COVID-19 (+) cases in the perioperative period and plan ahead patient management and protection of healthcare providers. Twenty-five to $30 \%$ of COVID-19 (+) cases worldwide occur in healthcare workers. It is for this reason that special personal protection equipment (PPE) must be used, together with usual contact and droplet precautions. Anesthesia procedures that involve airway management can produce infectious aerosol. In order to reduce risks, we make a series of recommendations on perioperative management of children at this time of pandemia, which are to be complemented with those already released by the Chilean Society of Anesthesiology (SACH).
\end{abstract}

\section{RESUMEN}

Desde diciembre de 2019 el mundo se ha enfrentado a la rápida extensión de un nuevo virus: el SARS-CoV-2. Originándose en Wuhan (China), se ha extendido a centenares de países, por lo que desde marzo de 2020 ya fue calificada como pandemia. Estamos frente a un virus nuevo de alta contagiosidad que ha llevado

\section{Key words:}

MeSH: pediatrics,

COVID-19, Anesthesia

\section{Palabras clave:}

pediatria, COVID-19, anestesia

\footnotetext{
Hospital de Carabineros de Chile, Santiago, Chile. Complejo Asistencial Sótero del Río Block Pediátrico. Santiago, Chile. Hospital Roberto del Río, Santiago, Chile. Clínica Alemana de Santiago. Santiago, Chile.

Hospital Carlos van Buren. Hospital Naval Almirante Nef, Universidad de Valparaíso. Valparaíso, Chile.

Hospital Roberto del Río, Santiago, Chile. Clínica Alemana de Santiago. Santiago, Chile.
}

\section{ORCID}

https://orcid.org/0000-0002-1212-7178

\section{Correspondencia:}

María Francisca Bernucci P.

Email: mbernucc@uc.cl 
al colapso de los sistemas sanitarios. Alrededor del 2\% de la población mundial de afectados son niños, los que en mayor frecuencia son asintomáticos, siendo relevantes en la transmisión de la enfermedad. En un bajo porcentaje pueden llegar a cuadros severos. En este artículo describimos la epidemiología pediátrica de COVID-19 reportada hasta ahora. Así, sabremos cuándo sospecharla en el perioperatorio y, por lo tanto, cómo manejar al paciente y proteger al personal de salud de contraer la infección. En este último punto, se ha recomendado el uso de elementos de protección personal (EPP) especiales y tomar precauciones de gotitas y contacto, ya que se conoce que entre el $25 \%$ y $30 \%$ de los contagiados en el mundo son trabajadores de la salud. En anestesia, el manejo de la vía aérea expone al contagio por aerosoles del virus. Por esta razón presentamos una serie de recomendaciones de manejo de niños en perioperatorio, las que son un complemento de las recomendaciones en población general publicadas ya por la Sociedad de Anestesiología de Chile (SACH).

\section{Introducción}

Desde diciembre de 2019 la enfermedad por SARS-CoV-2 (COVID-19) ha resultado ser un problema de salud a nivel mundial, con más de 1.776.867 casos y más de 111.828 muertes[1], siendo declarada pandemia por la Organización Mundial de la Salud el 11 de marzo de este año.

En este escenario se hace necesario consensuar protocolos adaptables para todos los centros hospitalarios que en la contingencia deberán manejar estos pacientes.

El objetivo de esta revisión es resumir la evidencia disponible sobre el comportamiento del virus en pacientes pediátricos y aportar con recomendaciones para el manejo de esta población en el perioperatorio.

Por ser un virus nuevo, la información existente es de baja o moderada calidad y está en dinámico desarrollo. Al momento de la redacción de este artículo la mejor evidencia disponible son guías de organismos internacionales y nacionales reconocidos, series de casos e informes epidemiológicos. En temas en que no hay evidencia suficiente, nos basamos en recomendaciones de expertos y/u opinión de las autoras.

\section{Epidemiología}

La literatura referente publicada a la fecha es escasa y señala en su mayoría a casos leves con recuperación íntegra. Incluso, los reportes de China, plantearon una "resistencia relativa" en niños[2].

Los primeros casos pediátricos reportados fueron hallazgos de estudios familiares, presentándose los pacientes como asintomáticos o con cuadros respiratorios menores[3]. No queda claro si en niños existe una real búsqueda activa en asintomáticos o si los pacientes reportados sólo han sido los que han presentado síntomas o tenían familiares positivos[4]. Esto resulta relevante ya que revela que cada país tiene distintos sistemas diagnósticos de detección, considerando positivos a pacientes con clínica sin realización de test diagnóstico[5]. Por esto resulta difícil lograr cifras comparativas y exactas de cuánta población pediátrica está afectada a la fecha o conocer los reales datos de la comorbilidad de los pacientes falleci$\operatorname{dos}[4],[5]$.

Según cifras norteamericanas entregadas por la Central de Control y Prevención de Enfermedades $(C D C)$, el 1,7\% ( $n=2.572)$ de los casos totales se encuentra entre 0-18 años, cifra similar a las reportadas en China que fluctúan entre un 1-2\% de la población total de infectados[6]-[8].

En el Gráfico 1, se presenta la distribución de casos por rango etario.

En los reportes que cuentan con información más detallada, el 23\% de los pacientes tenía al menos una comorbilidad, siendo las más frecuentes asma, patología cardíaca e inmunosupresión. Respecto a sexo, dos reportes describen una mayor tendencia en sexo masculino[6]-[8].

En Chile, el diagnóstico de COVID-19 requiere un resultado positivo en la reacción en cadena de la polimerasa con transcriptasa reversa en tiempo real (RT-PCR), de una muestra de secreción de la vía respiratoria[1].

Al 12 de abril de 2020, se registran 7.525 casos positivos en Chile, donde 425 casos corresponden a población entre 0-19 años. No existen datos de pacientes pediátricos hospitalizados o con necesidad de ventilación mecánica actualmente[1] (Gráfico 2). 


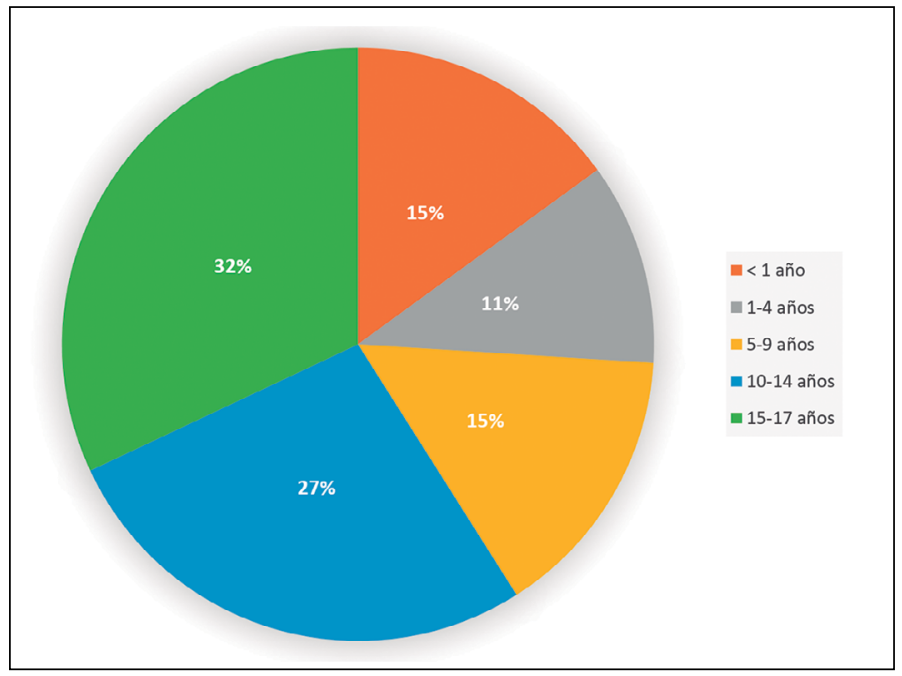

Gráfico 1. Incidencia según edad. EE. UU. CDC.

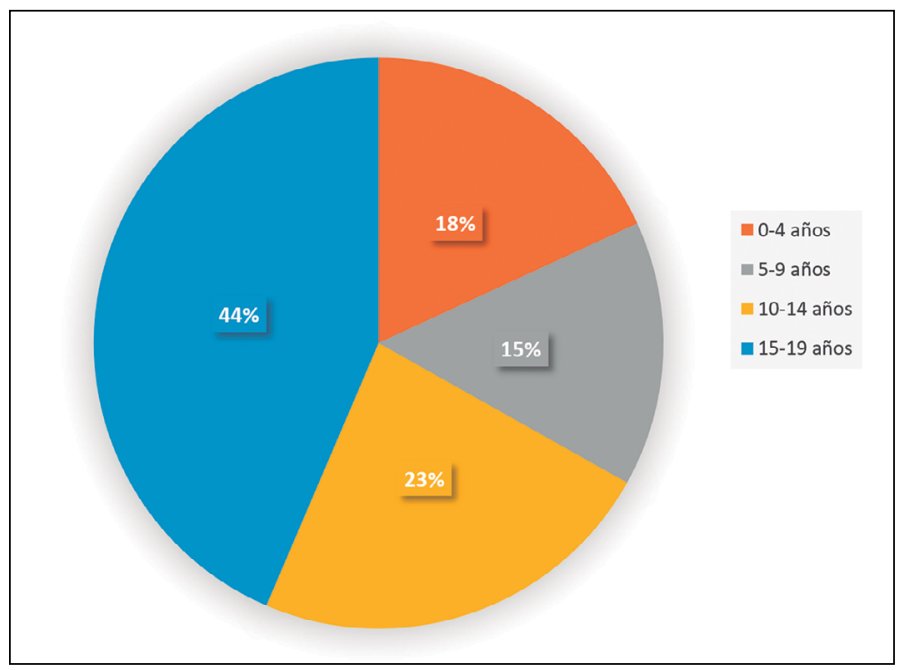

Gráfico 2. Incidencia según edad. Chile.

Respecto a la población neonatal, no hay a la fecha evidencia que exista transmisión directa de COVID-19 a neonatos por vía transplacentaria, canal del parto o lactancia materna. Sólo se tiene claridad que los casos reportados de infecciones a recién nacidos han sido a través del contagio por secreciones respiratorias de la madre en el postparto inmediato[9]-[11].

La Sociedad Chilena de Pediatría define recién nacido sospechoso, a aquel:

- Cuya madre tiene el antecedente de infección dentro de los 14 días previos y 28 días posteriores al parto.
- Que haya estado en contacto directo con algún infectado.

Por lo tanto, no es necesario que el neonato tenga síntomas para sospechar la infección[12].

\section{Cuadro clínico}

En la población pediátrica se ha observado frecuentemente la infección asintomática, detectada al estudiar contactos de casos o grupos familiares con miembros infectados. Cuando hay desarrollo de un 
cuadro clínico sintomático, generalmente corresponde a un cuadro respiratorio leve, que dura 1 a 2 semanas. Los pocos que progresan a infección respiratoria baja, generalmente son pacientes de subpoblaciones especiales: lactantes, pacientes con antecedentes de patología respiratoria o cardíaca subyacente e inmunocomprometidos [6],[13]-[15].

Las posibles presentaciones clínicas descritas son:

- Asintomática.

- Fiebre (53\%), tos seca (54\%), disnea (13\%), astenia. Estos síntomas se observaron de forma concomitante en un 73\%[6].

- Síntomas respiratorios altos (menos frecuentes): congestión nasal, rinorrea, faringitis.

- Otros síntomas respiratorios (también menos frecuentes): taquipnea, crépitos, retracción costal, cianosis[16].

- Síntomas gastrointestinales: dolor abdominal, náuseas, vómitos y diarrea.

Esta presentación clínica contrasta bastante con los adultos, quienes con mayor frecuencia tienen fiebre, tos y dificultad respiratoria. En los casos severos presentan disnea aproximadamente una semana posterior al inicio de los síntomas, progresando rápidamente a síndrome de distrés respiratorio del adulto (SDRA), shock séptico, acidosis metabólica refractaria y disfunción de la coagulación[14].

\section{Exámenes de laboratorio}

El recuento leucocitario es normal en la mayoría de los casos, aunque también se puede observar leucopenia y más infrecuente, leucocitosis. A nivel de subpoblaciones, puede haber linfopenia en un tercio de los casos y menos frecuentemente, linfocitosis.

Es común un incremento en los valores de creatinin kinasa $M B(C K-m b)$ y de proteína $C$ reactiva $(P C R)$. También es frecuente el aumento de la procalcitonina, distinto a lo que ocurre en adultos en quienes habitualmente se mantiene normal.

Puede haber aumento de mioglobinemia, velocidad de hemosedimentación (VHS) y con menor frecuencia, de la alanina aminotransferasa (ALT o SGPT). En casos severos, hay elevación de Dímero $D^{14}$.

En caso de pacientes críticos con falla multiorgánica, se alteran los marcadores de función de cada sistema fisiológico comprometido (respiratorio, cardíaco, renal, neurológico, hematológico)[17].

Las pruebas para estudiar la presencia del virus, como la RT-PCR, dan positivo con muestras biológicas de distintas zonas del cuerpo, como, por ejemplo, en hisopados nasofaríngeos y faríngeos; esputo y secreciones del tracto respiratorio bajo; deposiciones y sangre[14].

No es raro que el estudio de otros agentes infecciosos, como Influenza A y B, Mycoplasma, virus respiratorio sincicial (VRS) y citomegalovirus sean positivos, dado que la coinfección es muy frecuente[16].

\section{Exámenes de imagenología}

La radiografía de tórax puede ser normal, por lo que la tomografía axial computarizada (TAC) de pulmón puede aportar con mucha más información.

En etapas tempranas, en la TAC de tórax se ha observado desde pulmones sin alteraciones a lesiones pulmonares uni o bilaterales. También se han descrito lesiones subpleurales con inflamación localizada; opacidades en vidrio esmerilado pequeñas y cambios intersticiales en la periferia del parénquima pulmonar[16].

Si hay una evolución al deterioro, las lesiones observadas en etapas tempranas se intensifican y aumentan en número y distribución. De esta manera, se pueden apreciar opacidades múltiples en vidrio esmerilado bilaterales y/o sombras infiltrativas. Estas lesiones pueden coexistir con áreas de consolidación, signos de bronquiograma aéreo, lesiones fibrosas y engrosamiento septal interlobar. En casos severos, la consolidación pulmonar se hace mucho más extensa, abarcando ambos pulmones, dando la imagen de "pulmón blanco". Es raro que exista derrame pleural.

En el período de convalecencia, la mejoría radiológica es más lenta que la mejoría clínica[14],[16] (Figura 1).

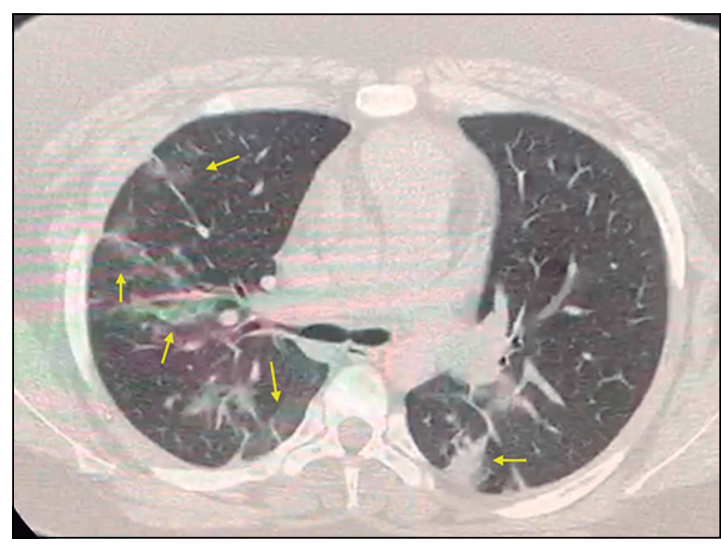

Figura 1. TAC de tórax de paciente de 13 años con insuficiencia respiratoria por COVID-19. Flechas muestran opacidades periféricas y centrales, principalmente a derecha. 


\section{Clasificación clínica}

\section{Infección asintomática}

El paciente tiene una prueba de detección positiva (según normativa local del país) sin tener manifestaciones clínicas ni cambios en exámenes imagenológicos.

\section{Infección respiratoria alta}

El paciente solo presenta fiebre, tos, odinofagia, congestión nasal, astenia, cefalea, mialgia, etc., sin presentar signos de neumonía en exámenes de imágenes ni sepsis.

\section{Neumonía leve}

El paciente tiene síntomas respiratorios como tos; puede tener o no fiebre; y su estudio de imágenes pulmonares es indicativo de neumonía, sin que alcance criterios de severa.

\section{Neumonía severa}

Se considera cuando se cumple cualquiera de los siguientes criterios:

- Taquipnea (valor frecuencia respiratoria ajustada por edad).

- SatHb $<92 \%$.

- Hipoxia evidenciada por ventilación accesoria (bostezo, aleteo nasal, retracción), cianosis, apnea intermitente.

- Alteración de conciencia: somnolencia, coma, convulsión.

- Rechazo alimentario, dificultad para alimentarse, signos de deshidratación.

\section{Caso crítico}

El que requiere ingresar a la Unidad de Cuidados Intensivos por falla respiratoria que requiere ventilación mecánica, shock o falla de otros órganos.

Se puede identificar de manera precoz a los posibles casos críticos, utilizando los criterios de manejo en niños de las neumonías adquiridas en la comunidad. De esta manera, los pacientes con mayor riesgo son los niños que presenten:

- Historia de contacto con casos severos de COVID-19.

- Condiciones clínicas preexistentes: cardiopatía congénita, hipoplasia broncopulmonar, anomalías del tracto respiratorio, niveles anormales de hemoglobina, malnutrición severa.

- Compromiso inmunitario.
Estos pacientes están en riesgo de evolucionar hacia un cuadro severo si presentan cualquiera de las siguientes situaciones:

- Disnea con taquipnea ajustada según edad, descartando efectos de llanto y fiebre.

- Fiebre persistente por 3-5 días.

- Compromiso del sensorio, letargia.

- Aumento de enzimas miocárdicas, hepáticas y lactato deshidrogenasa.

- Acidosis metabólica sin etiología.

- Imágenes de tórax con infiltrado bilateral o multilobar, derrame pleural o progresión rápida de esas condiciones.

- Lactantes menores de 3 meses.

- Complicaciones extrapulmonares.

- Coinfección con otros virus y/o bacterias ${ }^{14}$.

\section{Especificidad etaria de los casos}

La experiencia de estos meses de pandemia nos muestra que los niños de todas las edades son susceptibles de ser infectados. Sin embargo, tras exponerse al virus es menos probable que desarrollen la enfermedad, en comparación con los adultos. Hasta el momento, se han propuesto varias hipótesis para explicar esta situación:

- Menor riesgo de infección por menor exposición. Los niños, sobre todo los recién nacido y lactantes, suelen mantenerse en su hogar y es infrecuente que realicen viajes internacionales. En China, casi la totalidad de los casos pediátricos se dieron en grupos familiares, por contacto de un miembro adulto infectado con el niño.

- Menos comorbilidades en la población pediátrica, con un sistema respiratorio más sano por la poca o nula exposición a humo de cigarrillo y contaminación.

- Distinto grado de maduración fisiológica en los niños, modificando la interacción con el virus. En particular, distinta distribución y actividad de los receptores celulares utilizados por el virus para infectar.

- Respuesta inmune innata más activa en niños.

- "Imprinting" inmunológico, que consiste en que los adultos contarían con anticuerpos desarrollados por exposición previa a virus antigénicamente similares al virus del SARS-CoV-2. Estos anticuerpos podrían desencadenar la respuesta inmune aumentada y con ello un cuadro inflamatorio sistémico severo[2],[7],[13]. 


\section{Consideraciones del manejo perioperatorio}

En general, la atención perioperatoria del paciente pediátrico, sigue las recomendaciones de la Sociedad de Anestesiología de Chile para el manejo de pacientes con COVID-19[18]. A continuación, sólo detallaremos lo referente a la población pediátrica.

\section{Preoperatorio}

Como plan de acción ante la expansión de la infección por coronavirus, se ha implementado postergar las cirugías electivas, siempre que no implique un riesgo para el paciente. Para los casos que deban concurrir al centro quirúrgico, estos debiesen tener una valoración preclínica por el equipo para poder categorizar el riesgo.

Se recomienda realizar una encuesta epidemiológica al paciente y familiar directo, considerando las definiciones actuales de caso y contactos[19], lo que permitiría determinar el flujo del paciente dentro del hospital y las medidas de protección individual (EPP) a usar por el personal de salud. Esta encuesta se ha descrito vía remota[20], o presencial según el caso.

Se recomienda, generar flujos distintos para pacientes portadores de fiebre y síntomas de infección respiratoria, del resto de los pacientes[21], generando un sistema de triage interno.

\subsection{Evaluación clínica preoperatoria}

Dada la alta presencia de virus en la comunidad y que la mayoría de los niños se presentan asintomáticos o con síntomas inespecíficos respiratorios, debemos considerarlos a todos como potencial fuente de contagio. Así, ya desde la evaluación preoperatoria debemos mantener el distanciamiento social, lavado de manos antes y después de la atención, uso de EPP[18],[21],[22] y limpieza de superficies e implementos médicos (fonendoscopio, lápiz).

También se han descrito en este periodo evaluaciones preoperatorias vía teleconferencia u otro medio de comunicación remota, para intervenciones que no se pueden postergar[22].

Si bien la evaluación preoperatoria en el paciente pediátrico sigue los mismos acápites habituales, se deben considerar las siguientes precauciones para su desarrollo[22],[23]:

- Pensar que el contagio en general es por contacto previo en el círculo familiar en sus hogares, por tanto, reforzar en la entrevista la historia epidemiológica.

- El paciente debe ser acompañado por un sólo tutor (siempre la misma persona), el que no debe ser sospechoso ni positivo para COVID-19. Algunas recomendaciones incluyen un tutor menor de 65 años, no calificar como sospechoso y carecer de comorbilidades.

- Se debe minimizar el contacto cercano y mantener separación con otros pacientes de al menos 1,5 metros en todo momento[24].

- Se debe colocar mascarilla quirúrgica al paciente mayor de 2 años y a su tutor[20]. Tener precaución en menores de 2 años por el riesgo de sofocación.

- Se recomienda medir la temperatura y si ésta es mayor que $37,3^{\circ} \mathrm{C}$ el paciente debiera ser referido a la unidad de control de COVID del hospital.

- Los síntomas gastrointestinales de reciente inicio, pueden estar presentes. Es más, se deben consultar ya que este virus podría ser transmitido vía fecal-oral[25].

\subsection{Preparación preoperatoria[18],[23]}

- El acceso intravenoso en general se instala después de la inducción en pediatría. En estas condiciones se recomienda, de ser posible, instalarlo previo al ingreso a pabellón y siempre que las condiciones de seguridad y del paciente lo permitan.

- El control de la ansiedad preoperatoria podría disminuir el llanto y la generación de aerosoles, por lo que podría individualizarse su indicación según el caso. Para premedicación, privilegiar vía oral o intravenosa y evitar la vía nasal[21].

- En pacientes con hiperreactividad bronquial, se recomienda uso de inhalador de dosis medida con aerocámara, para la premedicación con salbutamol. Evitar el uso de nebulizaciones.

- Dado que se debe restringir el número de personas que ingresan a pabellón, la inducción con presencia parental no sería recomendable.

\section{Intraoperatorio[18],[23]}

El flujo de presión positiva del ambiente de pabellón es un riesgo de dispersión viral, sumado a procedimientos de alto riesgo de generar aerosoles (intubación, aspiración, extubación, reanimación cardiopulmonar y terapias respiratorias). Esto llama a extremar las medidas de planificación y ejecución de las técnicas anestésicas.

\subsection{Respecto al pabellón}

- Disponer de una lista de chequeo anestésico (vía aérea, drogas, EPP, roles, monitorización) y quirúrgico previo al ingreso del paciente a pabellón.

- Mantener uso correcto de EPP. 
- Uso de circuito respiratorio con filtros. Éstos deben ser antimicrobianos de alta eficiencia HMEF (Heat and Moisture Exchanger Filter), que tienen un filtro integrado.

- Se recomienda posicionar un filtro en la rama espiratoria del circuito y otro entre el tubo endotraqueal y la pieza en $Y$.

- Es preciso considerar las especificaciones técnicas de cada filtro, ya que estos tienen un efecto directo sobre la resistencia y el espacio muerto. Se debe revisar el rango de volumen corriente para cada filtro en que se debe utilizar en forma segura, fuera del cual se reduce su eficiencia en relación a la resistencia y reinhalación. Considerar el peso del filtro ya que este puede incrementar con la condensación, lo que generará mayor tracción sobre el circuito[26].

- Especial atención merecen los pacientes menores de $5 \mathrm{~kg}$, con un volumen corriente entre 3 a $30 \mathrm{ml}$, en quienes se debe extremar los cuidados para no aumentar el espacio muerto[18].

- Se recomienda cambiar los filtros luego de 3 o 4 horas de anestesia.

- Para la línea de capnografía, la toma de muestra de aire debe pasar por el filtro HMEF. Hasta ahora no hay evidencia sobre el uso de otros filtros, por ejemplo, los peridurales, para la protección del módulo de capnografía.

\subsection{Manejo anestésico}

- Durante la preoxigenación se recomienda cubrir nariz y boca, generando un buen sello. Esta se debe realizar con $\mathrm{FIO}_{2}$ al $100 \%$ por 5 minutos. En lo posible colocar la mesa quirúrgica semisentada $\left(30^{\circ}\right)$.

- El flujo de gas fresco debe ser menor a $6 \mathrm{~L}$ $\min [22]$.

- En pacientes con baja reserva, los sistemas de alto flujo podrían usarse para aumentar la tolerancia a la apnea en inducción de secuencia rápida. Si bien estos sistemas se desaconsejaban en un inicio, se pueden considerar en pabellones con presión negativa y usando las medidas de seguridad del personal[24],[29],[31],[32].

- Se debe privilegiar la inducción endovenosa, que limitaría la aerosolización.

- Para pacientes sin acceso venoso o que lo ha perdido en el traslado, intentar según la edad instalarla en pabellón o realizar inducción inhalatoria a dosis iniciales altas de agente inhalatorio para asegurar una apnea rápida. Con un anestesiólogo exclusivo manejando la vía aérea (a dos manos) y un segundo operador que maneja la bolsa de ventilación, vaporizador, los parámetros ventilatorios y el resto de la máquina de anestesia.

- En pacientes adultos COVID-19 se recomienda inducción de secuencia rápida. En niños pequeños, esta inducción es difícil de realizar dada la poca reserva funcional y aumentada tasa de desaturación. Por lo que se utiliza una inducción de secuencia rápida modificada, realizando una ventilación gentil a no más de $12 \mathrm{cmH}_{2} \mathrm{O}$ [27].

- No existe la evidencia para recomendar algún inductor endovenoso en particular por sobre otro.

- Se recomienda como primera opción el uso de rocuronio 1,2 mg/kg, antes que succinlicolina (dosis de $1,5 \mathrm{mg} / \mathrm{kg}$ ).

- Las medidas de aislamiento físico para el manejo de la vía aérea como: material plástico adosado a la mascarilla, sábana plástica que cubre cabeza y cara, caja para intubación de policarbonato, se han usado en diferentes centros y se pueden considerar.

Un reciente artículo[28] muestra una caja para cubrir la cabeza del paciente con dos puertos circulares para introducir las manos, la que fue probada con simulación y pareciera que restringiría la dispersión de aerosoles, como una medida adicional de aislamiento. Pero debemos considerar el entrenamiento previo que se debe adquirir antes del uso con pacientes, ya que restringe el movimiento de las manos y se debe considerar el tamaño del dispositivo según la edad del paciente.

- Para la intubación oral, al igual que en los adultos, se recomienda realizar con videolaringoscopio si es que tiene el entrenamiento previo[29], con el fin de aumentar la distancia del operador a la vía aérea.

- En caso de utilizar laringosocopía directa, se debe poner especial atención para evitar episodios de tos.

- Para el manejo de la vía aérea, se recomienda uso de tubos endotraqueales con balón para lactantes mayores de $3 \mathrm{Kg}$, el que debe ser inflado inmediatamente luego de la intubación para evitar la fuga de aire[24],[30]. Posteriormente, se debe medir la presión del balón para que no supere los 20 $\mathrm{cmH}_{2} \mathrm{O}$, dadas las características anatómicas de la vía aérea del niño.

- Para la correcta posición del tubo endotraqueal, la auscultación tiene un rol limitado en pacientes con SDRA severo, además del riesgo de contaminación del equipo, del staff y de las dificultades 
técnicas por los EPP[29]. Se deberán usar los otros medios de confirmación.

- Evitar el uso de dispositivos supraglóticos (DSG) como primera opción para manejo intraoperatorio. Sin embargo, se debe considerar su uso precozmente para la oxigenación[29],[30].

- Si se utiliza un DSG se recomienda uso de ventilación espontánea en vez de ventilación controlada para evitar mayor fuga[29].

- Evitar las desconexiones del circuito al ventilador y clampear el tubo endotraqueal cuando ésto es requerido con el filtro puesto cercano al paciente. Pause el ventilador cuando requiera desconectar el circuito[22].

- Para la extubación se recomienda minimizar los accesos de tos y la exposición a secreciones. Considerar medidas físicas de aislamiento de aerosoles y/o el uso de dexmedetomidina, lidocaína u opioides[29]. Esto, además, ayudaría a evitar otra posible causa de aerosolización del virus en el pabellón: la agitación del despertar.

- Considerar profilaxis máxima de náuseas y vómitos postoperatorios para evitar aerosolización en el postoperatorio.

- La aspiración de secreciones se debe limitar a las imprescindibles[24].

- Se recomienda un sistema cerrado de succión para reducir la producción de aerosoles virales, para pacientes que continuarán con ventilación mecánica[24]. Idealmente éste debiese ser instalado en el momento de la intubación para minimizar intervenciones posteriores[29].

- La anestesia neuroaxial y bloqueos locoregionales están recomendados por ser técnicas que no generan aerosoles y contribuyen a la analgesia. Se debe mantener la mascarilla quirúrgica en el paciente, para pacientes con regional única[18].

\section{Postoperatorio}

- Se recomienda evitar trasladar pacientes a la Unidad de Recuperación Postanestésica para limitar la dispersión del virus. Para ello, sugerimos hacer recuperación en el mismo pabellón antes de trasladar a pieza (de aislamiento). Si su unidad de destino es una Unidad de Cuidados Intermedios o UCl, se puede trasladar inmediatamente postanestesia (según normas locales)[18].

- Después de la extubación, el paciente debiese usar mascarilla quirúrgica simple en todo momento.

- Podría usarse nariceras en niños pequeños con flujos bajos (0,2 L/min/kg, máximo $6 \mathrm{~L} / \mathrm{min})$, que podrían ser mejor toleradas.
- Si se debe mantener intubado se puede usar un Ambú ${ }^{\circledR}$ de uso individual para el paciente en el traslado a $\mathrm{UCl}$, con el filtro (HMEF) entre mascarilla y dispositivo de ventilación o su ventilador de transporte.

\section{Consideraciones para manejo de vía aérea fuera del pabellón}

De requerirse manejo de vía aérea fuera de pabellón ésta requiere una planificación detallada y una técnica cuidadosa por la alta carga viral y por ser un procedimiento generador de aerosoles[31]. Se debe privilegiar la intubación orotraqueal y se debe verificar disponibilidad de videolaringoscopio. Debe ser ejecutado por un médico experto para optimizar que sea lograda al primer intento[29]; pudiese ser un anestesiólogo experimentado asistido por otro médico.

Se recomienda mantener una comunicación fluida con los equipos de urgencia y cuidados críticos para anticipar la necesidad de intubación de pacientes graves, en los cuales está recomendada una intubación temprana[20]. La preparación del equipo incluye:

- El uso de EPP.

- Lista de chequeo de equipos de:

- Vía aérea de primera línea y de rescate.

- Drogas.

- Un equipo entrenado y con roles definidos.

- Delimitar áreas de trabajo[20].

Considerar que la ventilación con bolsa-mascarilla debe ser hecha con un buen ajuste facial y con mínima presión, sin hiperventilar y evitando fugas[31]. El uso de filtro HMEF debe ser instalado entre la mascarilla y el circuito respiratorio o resucitador manual.

\section{Consideraciones para el manejo de la reanimación cardiopulmonar en pacientes pediátricos con sospecha o confirmación de COVID-19}

Diversas recomendaciones de las organizaciones internacionales han sido adaptadas para atención de pacientes COVID-19 [33],[34]. En relación a éstas, se destaca como principios generales:

- Se debe reducir la exposición del personal, lo que implica:

- Detección precoz de casos gravemente enfermos, quienes tienen riesgo de deterioro agudo o paro cardiorrespiratorio, para poder prevenir el atender la reanimación sin los EPP. 
- El EPP debe ser instalado antes de iniciar maniobras (escudo facial, mascarilla N95, bata y guantes).

- Limitar en número de integrantes de la reanimación a lo estrictamente necesario.

- Las maniobras debieran realizarse en salas aisladas.

- Las recomendaciones de los algoritmos se mantiene.

En pediatría, la causa más probable sigue siendo la respiratoria, lo que hace que las ventilaciones sean cruciales. Debe estar disponible de inmediato para cualquier paciente de riesgo el equipo de ventilación con bolsa, mascarilla e intubación, con filtros de alta eficiencia.

\section{Conclusiones}

El virus SARS-CoV-2 nos plantea un gran desafío por delante. Dadas las características epidemiológicas y su rápido contagio, cobra mayor importancia en la población pediátrica las acciones preventivas en el control de aislamiento y medidas generales de cuidado. Es importante cuidar al personal de salud, por esto reforzar el correcto uso de EPP y el entrenamiento por medio de simulación, son fundamentales.

Todos los niños son susceptibles de contraer el virus, si bien en general presentan cuadros leves y un bajo porcentaje requiere $\mathrm{UCl}$, existe riesgo de tener cuadros graves y mortalidad asociada.

Las decisiones clínicas seguirán cambiando a medida que conozcamos más del comportamiento de este virus. Por tanto, la recopilación de información a nivel nacional e internacional es perentoria para avanzar en el conocimiento de esta enfermedad.

\section{Fuentes de Financiamiento}

No se requirieron ni utilizaron.

\section{Conflictos de Interés}

Ninguno.

\section{Referencias}

1. (MINSAL) MdSdC. Informe Epidemiológico Enfermedad por SARS-CoV-2 (COVID-19). Chile 13-04-2020. In: Epidemiología D, editor. Santiago, Chile; 2020.

2. Lee $\mathrm{PI}, \mathrm{Hu} Y \mathrm{~L}, \mathrm{Chen} P Y$, Huang YC, Hsueh PR. Are children less susceptible to COVID-19? J Microbiol Immunol Infect. 2020 Feb;S1684-1182(20)30039-6. PMID:32147409

3. Pan X, Chen D, Xia Y, Wu X, Li $T$, Ou $X$, et al. Asymptomatic cases in a family cluster with SARS-CoV-2 infection. Lancet Infect Dis. 2020 Apr;20(4):4101. https://doi.org/10.1016/ S1473-3099(20)30114-6 PMID:32087116

4. Cao Q, Chen YC, Chen CL, Chiu $\mathrm{CH}$. SARS-CoV-2 infection in children: transmission dynamics and clinical characteristics. J Formos Med Assoc. 2020 Mar;119(3):670-3. https://doi. org/10.1016/j.jfma.2020.02.009
PMID:32139299

5. Dong $Y$, Mo X, Hu Y, Qi X, Jiang $F$, Jiang $Z$, et al. Epidemiology of COVID-19 Among Children in China. Pediatrics. 2020 Mar;145(6):e20200702. https:// doi.org/10.1542/peds.20200702 PMID:32179660

6. Bialek S, Gierke R, Hughes M, McNamara LA, Pilishvili T, Skoff T; CDC COVID-19 Response Team. Coronavirus Disease 2019 in Children - United States, February 12-April 2, 2020. MMWR Morb Mortal Wkly Rep. 2020 Apr;69(14):422-6. https://doi.org/10.15585/mmwr. mm6914e4 PMID:32271728

7. Mizumoto $K$, Omori R, Nishiura $H$. Age specificity of cases and attack rate of novel coronavirus disease (COVID-19). Cold Spring Harbor Laboratory; 2020 Mar 13; Available from: http://dx.doi. org/10.1101/2020.03.09.20033 142

8. Dong $Y$, Mo X, Hu Y, Qi X, Jiang $F$, Jiang $Z$, et al. Epidemiology of COVID-19 Among Children in China. Pediatrics [Internet]. American Academy of Pediatrics (AAP); 2020 Mar 16;e20200702. https://doi. org/10.1542/peds.2020-0702 PMID:32179660

9. Chen H, Guo J, Wang $C$, Luo F, Yu X, Zhang W, et al. Clinical characteristics and intrauterine vertical transmission potential of COVID-19 infection in nine pregnant women: a retrospective review of medical records. Lancet. 2020 Mar;395(10226):809-15. https://doi.org/10.1016/ S0140-6736(20)30360-3 PMID:32151335

10. Davanzo R, Mosca F, Moro G, Santi F. BREASTFEEDING and SARS-COV-2 INFECTION (Coronavirus Disease 2019 COVID-19). 2020:1-7.

11. CDC. Interim Guidance on Breastfeeding for a Mother Confirmed or Under Investigation For COVID-19. In: 
CDC, editor. NIRD) NCflaRD, Disease DoV. 2020.

12. Pediatría SCd. Recomendaciones para la prevención y manejo del recién nacido. In: Neonatología $\mathrm{Rd}$, Infectología Rd, Nutrición $\mathrm{Rd}$, Nutrición Rd, Lactancia $\mathrm{Cd}$, Neonatales CCdl, editors. Pandemia COVID-19: Sociedad Chilena de Pediatría; 2020. p. 1-10.

13. Wei M, Yuan J, Liu Y, Fu T, Yu $X$, Zhang ZJ. Novel Coronavirus Infection in Hospitalized Infants Under 1 Year of Age in China. JAMA. 2020 Feb;313(13):1313-4. https://doi. org/10.1001/jama.2020.2131 PMID:32058570

14. Shen $K$, Yang $Y$, Wang $T$, Zhao $D$, Jiang $Y$, et al. Diagnosis, treatment, and prevention of 2019 novel coronavirus infection in children: experts' consensus statement. World Journal of Pediatrics. Springer Science and Business Media LLC; 2020 Feb 7; https://doi.org/10.1007/s12519020-00343-7 PMID:32034659

15. Cruz AT, Zeichner SL. COVID-19 in Children: Initial Characterization of the Pediatric Disease. Pediatrics. 2020 Mar;e20200834. https://doi. org/10.1542/peds.2020-0834 PMID:32179659

16. Xia W, Shao J, Guo Y, Peng $X$, Li Z, Hu D. Clinical and CT features in pediatric patients with COVID-19 infection: different points from adults. Pediatr Pulmonol. 2020 May;55(5):1169-74. https:// doi.org/10.1002/ppul.24718 PMID:32134205

17. Sun $D$, Li $H$, Lu XX, Xiao $H$, Ren J, Zhang FR, et al. Clinical features of severe pediatric patients with coronavirus disease 2019 in Wuhan: a single center's observational study. World J Pediatr. 2020 Mar. https://doi.org/10.1007/s12519-
020-00354-4 PMID:32193831

18. Recomendaciones para el manejo de pacientes con COVID19 en el perioperatorio. Revista Chilena de Anestesia. Asociacion de Medicos Anestesiologos de Chile; 2020 Mar 31;49(2):196-202. http://dx.doi.org/10.25237/ revchilanestv49n02.03.

19. Chile MdSd. Actualización de definición de caso sospechoso para vigilancia epidemiológica ante brote COVID-19. In: Pública. SdS, Asistenciales SdR, editors. www.minsal.cl 2020.

20. Thampi S, Yap A, Lijia F, Ong J. Special considerations for the management of COVID-19 pediatric patients in the operating room and pediatric intensive care unit in a tertiary hospital in Singapore. Pediatric Anesthesia.n/a(n/a).

21. Reanimación SEdAy. Documento Consenso. Recomendaciones Anestesia Pediátrica. Anestesia Pediátrica; 2020. pp. 1-8.

22. Francophone AdA-RPdE. Protocole de prise en charge des patients pédiatriques en période d'épidémie de COVID+ hors cas confirmés COVID+. 2020.

23. Chen X, Liu Y, Gong Y, Guo $X$, Zuo M, Li J, et al.; Chinese Society of Anesthesiology, Chinese Association of Anesthesiologists. Perioperative Management of Patients Infected with the Novel Coronavirus: Recommendation from the Joint Task Force of the Chinese Society of Anesthesiology and the Chinese Association of Anesthesiologists. Anesthesiology. 2020 Mar;1. https://doi.org/10.1097/ ALN.0000000000003301 PMID:32195699

24. Calvo Cea. Recomendaciones sobre el manejo clínico de la infección por el «nuevo coronavirus» SARS-CoV2. Grupo de trabajo de la Asociación Española de Pediatría (AEP). An Pediatr. Barc; 2020.

25. Yeo C, Kaushal S, Yeo D. Enteric involvement of coronaviruses: is faecal-oral transmission of SARS-CoV-2 possible? Lancet Gastroenterol Hepatol. 2020 Apr;5(4):335-7. https://doi.org/10.1016/ S2468-1253(20)30048-0 PMID:32087098

26. S F. E G, GP,R R-LM. Utilización de filtros bacterianos/virales durante la ventilación mecánica invasiva. Med Intensiva. 2013;30(1).

27. Engelhardt T. Rapid sequence induction has no use in pediatric anesthesia. Paediatr Anaesth. 2015 Jan;25(1):5-8. https:// doi.org/10.1111/pan.12544 PMID:25265988

28. Canelli R, Connor CW, Gonzalez M, Nozari A, Ortega R. Barrier Enclosure during Endotracheal Intubation. N Engl J Med. 2020 Apr;NEJMc2007589. https://doi. org/10.1056/NEJMc2007589 PMID:32243118

29. Cook TM, El-Boghdadly K, McGuire B, McNarry AF, Patel A, Higgs $A$. Consensus guidelines for managing the airway in patients with COVID-19: Guidelines from the Difficult Airway Society, the Association of Anaesthetists the Intensive Care Society, the Faculty of Intensive Care Medicine and the Royal College of Anaesthetists. Anaesthesia. 2020 Mar. https:// doi.org/10.1111/anae.15054 PMID:32221970

30. Cheung JC, Ho LT, Cheng JV, Cham EY, Lam KN. Staff safety during emergency airway management for COVID-19 in Hong Kong. Lancet Respir Med. 2020 Apr;8(4):e19. https://doi.org/10.1016/ S2213-2600(20)30084-9 PMID:32105633 
31. Wax RS, Christian MD. Practical recommendations for critical care and anesthesiology teams caring for novel coronavirus (2019-nCoV) patients. Can J Anaesth. 2020 Feb;67(5):56876. https://doi.org/10.1007/ s12630-020-01591-x PMID:32052373

32. Greenland JR, Michelow MD,
Wang L, London MJ. COVID-19 Infection: Implications for Perioperative and Critical Care Physicians. Anesthesiology. 2020 Mar;1. https://doi.org/10.1097/ ALN.0000000000003303 PMID:32195698

33. Association $\mathrm{AH}$. Interim Guidance for Healthcare Providers during COVID-19
Outbreak: American Heart Association; 2020. https:// professional.heart.org/idc/ groups/ahamah-public/@ wcm/@sop/@smd/documents/ downloadable/ucm_505872.pdf

34. UK RC. Resuscitation Council UK Statement on COVID-19 in relation to CPR and resuscitation in Paediatrics. 2020 04-03-2020 